\title{
EFFECT OF ALGAE TREATMENT ON STEVIA REBAUDIANA GROWTH
}

\author{
RÉKA CZINKÓCZKY1 AND Áron NÉMETH *1 \\ ${ }^{1}$ Department of Applied Biotechnology and Food Science, Budapest University of Technology and \\ Economics, Múegyetem rkp. 3, Budapest, 1111, HUNGARY
}

\begin{abstract}
Stevia rebaudiana Bertoni is a small, perennial and herbaceous shrub which originated in Paraguay (South America). Stevia rebaudiana is not native to Hungary but its cultivation and consumption may have many benefits, e.g. to reduce blood pressure and as a non-caloric sweetener. Steviol glycosides, mostly stevioside and rebaudioside A, located in the leaves are about 200-300 times sweeter than sucrose. S. rebaudina cultivation in Hungary would offer many opportunities in healthcare and the sweet industry. With the aim of achieving good green biomass yields, the effect of MACC4 autotrophic and heterotrophic algae strains was investigated by testing them as both leaf and soil fertilizers in the soil of Stevia rebaudiana seedlings and in its aqueous rooting experiments. In one of the later set up, the formation of roots was improved by combining the application of red light and algae treatment.
\end{abstract}

Keywords: Stevia rebaudiana, Steviol glycosides, algae treatment, Chlorella vulgaris

\section{Introduction}

Stevia rebaudiana Bertoni (Fig. 1) is a perennial shrub and a member of the Asteraceae family. Stevia originated in Brazil and Paraguay (South America). This plant is widely used by the Guaraní Indians of South America to sweeten tea $[1,2]$. S. rebaudiana was botanically classified in 1899 by Moisés Santiago Bertoni, who described it in more detail. Initially called Eupatorium rebaudianum, its name changed to $S$. rebaudiana (Bertoni) in 1905.

The sweet principle was first isolated in 1909 and only in 1931 was the extract purified to produce stevioside, its chemical structure was established in 1952 as a diterpene glycoside. Stevioside (Fig. 2) is described as a glycoside comprised of three glucose molecules attached to an aglycone referred to as steviol moiety $[3,4]$. S. rebaudiana also has other names like Sweet leaf, Sweet Herb of Paraguay, Sweet Honey Leaf and candyleaf. The sweetening components of the plant, i.e. steviol glycosides, were described in 1931 [5]. S. rebaudiana and its extracts have been used for a long time in Asia, South America and several countries in Europe. S. rebaudiana leaves and highly refined extracts are used as low-calorie sweeteners in Korea, Japan and Brazil [6]. Stevioside, one of the steviol glycosides, has been reported to lower the postprandial blood glucose concentration of Type II diabetics and the blood pressure of mildly hypertensive patients [7]. S. rebaudiana is used by diabetics as a diet therapy, and its extracts exhibit pharmacological effects such as anti-insulin resistance, the promotion of insulin secretion, as well as antihypertensive and anti-obesity proper-

\footnotetext{
*Correspondence: naron@f-labor.mkt.bme.hu
}

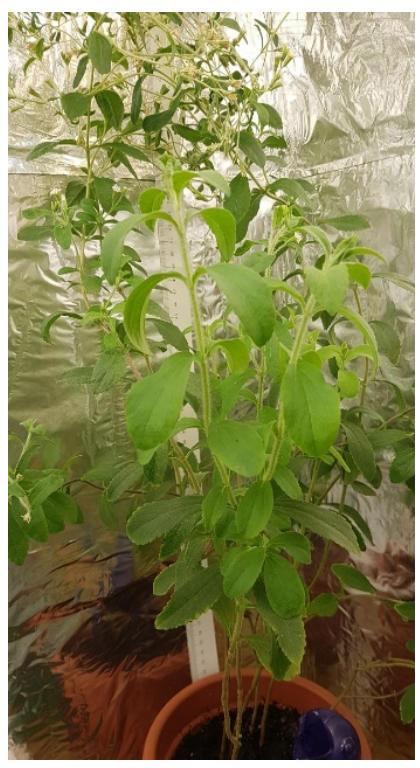

Figure 1: Stevia rebaudiana

ties [8]. Nowadays, the utilization of alternative sweeteners has become a viable option for producing low- or zero-calorie foods due to the development of the healthy food industry which intends to reduce the sucrose content of food products by the total or partial replacement of sucrose with alternative sweeteners.

Steviol glycosides are mainly produced in the leaves of the plant. The major components are steviol, stevioside and rebaudioside $\mathrm{A}$. The typical proportions of the major components of the leaves are stevioside (5-10\% of the 


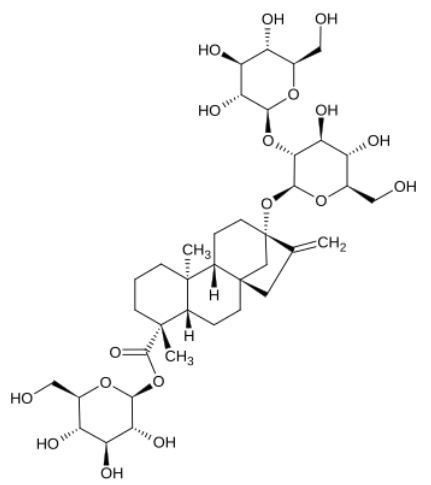

Figure 2: Structure of stevioside

total dry weight of the leaves), Reb A (2-4\%), Reb C (1-2\%) and dulcoside A (0.4-0.7\%) [9].

The leaves of $S$. rebaudiana are sessile, $3-4 \mathrm{~cm}$ in length, elongate-lanceolate or spatulate in shape with blunt-tipped laminae in addition to serrated margins from the middle to the tip and on the entire underside. The upper surface of the leaves is slightly glandular-pubescent. The stem is weak-pubescent at its base and woody. The rhizome has slightly branching roots. Flowers are composite surrounded by an involucre of epicalyx. The capitula are in loose, irregular, sympodial cymes. The flowers (Fig. 3) are white and pentamerous [5].

This plant can grow up to $1 \mathrm{~m}$ tall if it is exposed to sufficient light and receives enough nutrients as well as water. Therefore, it is worth considering the examination of crop production due to its wide range of applications.

Chlorella vulgaris is a eukaryotic unicellular green algae which is one of the fastest growing microalgae. This algae can be used in biodiesel processing following cell cultivation. The economic feasibility of algal biodiesel production highly depends on the biomass productivity and lipid yield [10]. Green algae (like Chlorella vulgaris) may produce phytohormones which can influence the growth of plants. Odgerel et al. used Chlorella vulgaris as a biofertilizer on barley and wheat. Faheed et al. used C. vulgaris as a biofertilizer on lettuce plants. These results showed that algae treatment yields longer roots and shoots of wheat compared to control $[11,12]$.

The aim of our work was to test Chlorella vulgaris

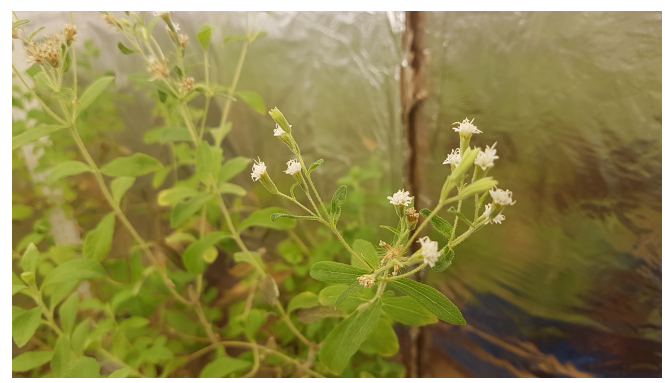

Figure 3: Stevia rebaudiana flowers

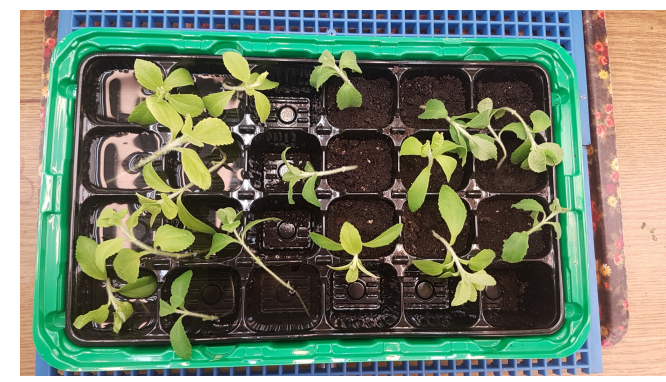

Figure 4: Plant cells

MACC4 autotrophic and heterotrophic cultures of microalgae to enhance the roots, biomass and stems of $S$. rebaudiana.

\section{Materials and methods}

In our work, the effects of hormones produced by algae on $S$. rebaudiana were tested. Phytohormones may improve its development. The plants were in a phytotron $\left(25 \pm 1{ }^{\circ} \mathrm{C}\right)$ where they received 16 hours of light per day. Each plant was placed in a $4.5 \mathrm{~cm} \times 5.0 \mathrm{~cm}$ planting cell (Fig. 4).

In Table 1, the blue background denotes the rootgrowing experiments where seedlings were placed into water as a control and into an aqueous algal suspension for trials. Furthermore, the green background indicates the modeling of experiments in land: while seedlings in samples of commercial potting soil were sprinkled with water as a control and an algal suspension as to test changes in biomass, the growth of roots and stems was recorded in terms of weight and length, respectively. Cells were replicated three times for each setting.

As algal suspensions, Chlorella vulgaris MACC4 heterotrophic and autotrophic cultivated strains were used. The cell suspension was diluted by up to 300 times with water to achieve a concentration of $5 \times 10^{7}$ colonyforming units (CFU)/ml. From this solution, $2 \mathrm{ml}$ was sprinkled onto each cell every 2 to 3 days.

The duration of the experiments was about five weeks. At the end of the fifth week (Fig. 5), the mass of the total green biomass and the length of the stem and roots in both water and soil were measured. For the results a statistical evaluation was conducted with Minitab 17 statistical software. The statistical analysis consisted of a two-sample

Table 1: Planting cells (H: heterotrophic, A: autotrophic, $\mathrm{C}$ : control, Green: seedlings in soil, Blue: seedlings in water, White: empty cells)

\begin{tabular}{|c|c|c|c|c|c|}
\hline $\mathrm{H}$ & $\overline{5 . C}$ & 9. - & 13. $\mathrm{H}$ & 17. C & $21 . \mathrm{A}$ \\
\hline $\mathrm{H}$ & $\overline{6 . C}$ & 10. - & 14. $\mathrm{H}$ & $18 . \mathrm{C}$ & \\
\hline & 7. C & 11. - & 15. $\mathrm{H}$ & 19. C & \\
\hline & 8. A & 12. A & 16. & 20. - & \\
\hline
\end{tabular}




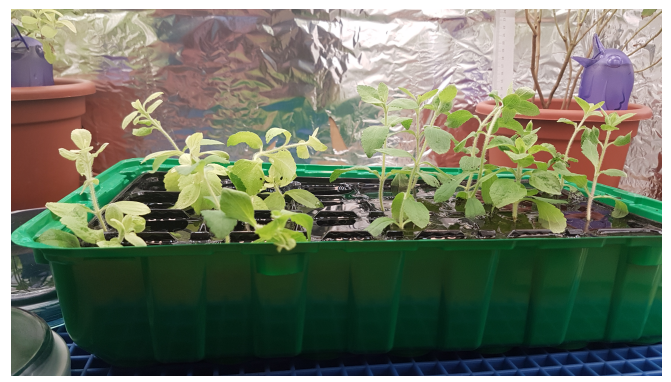

Figure 5: S. rebaudiana plants at the end of the experiment

t-test where the algae-treatment samples were compared against the controls and each other.

\section{Results and discussion}

The two-sample t-test in terms of the growth in biomass observed in the seedlings planted in soil is presented in Fig. 6. The p-value shows whether or not the treatment had an effect on biomass growth.

The comparison between heterotrophic algae treatment and the control samples is presented in Fig. 6A. The p-value was 0.653 , i.e. no significant difference exists between the two groups of results that were examined. A

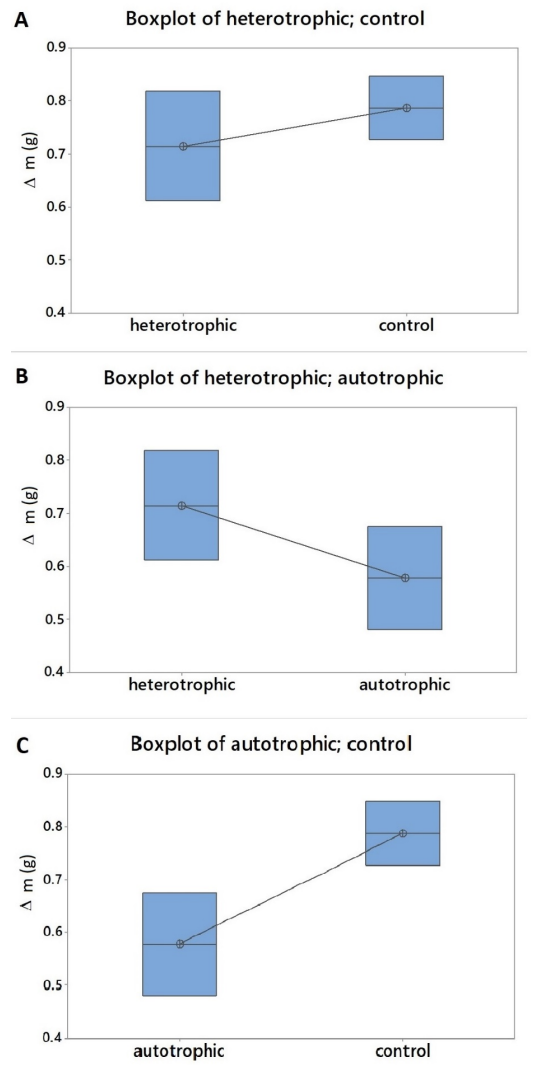

Figure 6: Box plots of two-sample t-tests: biomass growth in soil (A: heterotrophic algae vs. control, B: heterotrophic vs. autotrophic algae, C: autotrophic algae vs. control). comparison between the heterotrophic and autrotrophic algae cultures is presented in Fig. 6B. The p-value was 0.511 , i.e. no significant difference exists between them. A contrast is made between the autotrophic algae treatment and the control samples in Fig. 6C. The p-value was 0.316 , i.e. no significant difference exists between these groups either. From the results it can be seen that algae treatment does not have a positive effect on the growth of the green biomass of $S$. rebaudiana with regard to model experiments in soil.

The two-sample t-tests in terms of stem growth are presented in Fig. 7. A comparison between the heterotrophic algae treatment and the control samples is presented in Fig. 7A. The p-value was 0.642 , i.e. no significant difference exists between them. The results of heterotrophic versus autotrophic algae treatment cultures are shown in Fig. 7B. The p-value was 0.055 , i.e. once more no significant difference exists between them. A contrast between autotrophic algae treatment and control samples is presented in Fig. 7C. The p-value was 0.147 , i.e. yet again no significant difference exists between both groups. From these results it can be concluded that no significant improvements were observed in terms of the yield of plant biomass of algae treatments applied to seedlings planted in soil. However, the autotrophic al-

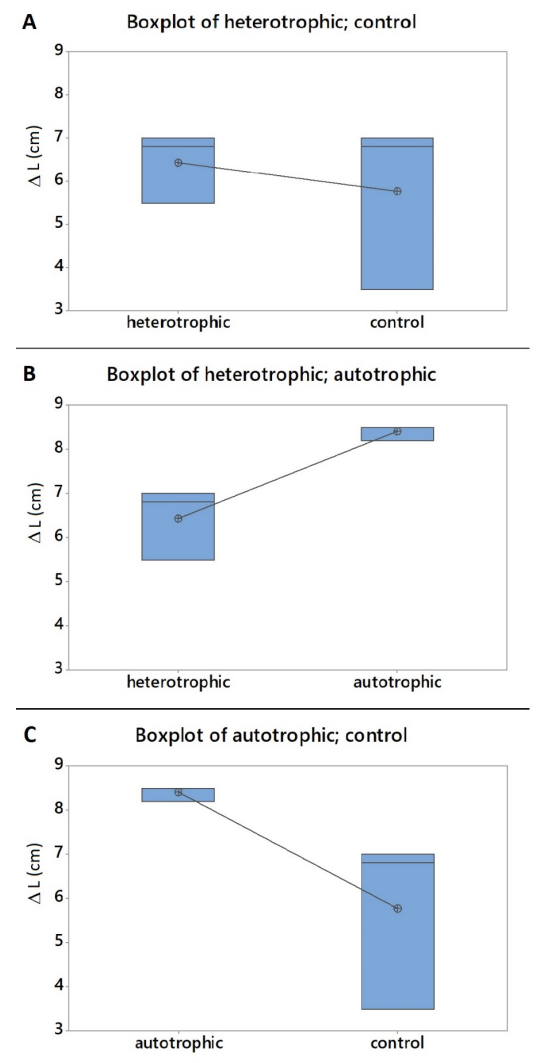

Figure 7: Box plots of two-sample t-tests in terms of stem growth in soil: (A: heterotrophic algae vs. control, B: heterotrophic vs. autotrophic algae, C: autotrophic algae vs. control) 


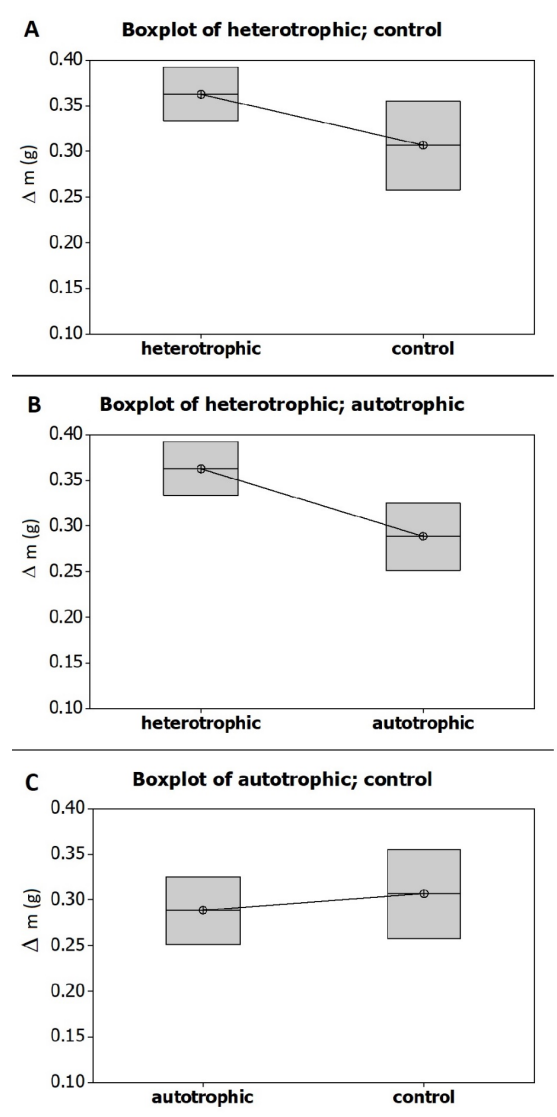

Figure 8: Box plots of two-sample t-tests in terms of root growth in land (A: heterotrophic algae vs. control, B: heterotrophic vs. autotrophic algae, C: autotrophic algae vs. control)

gae treatment seemed to be the most significant which suggests a weak positive effect in terms of the plant development of $S$. rebaudiana in soil may occur.

The two-sample t-tests in terms of the root growth observed in soil are presented in Fig.8. A comparison between heterotrophic algae treatment and the control samples is presented in Fig. 8A. The p-value was 0.503, i.e. no significant difference exists between them. A contrast between the heterotrophic and autotrophic algae treatments is shown in Fig. 8B. The p-value was 0.357, i.e. no significant difference exists between them either. The difference between autotrophic algae treatment and the control samples is presented in Fig. $8 \mathrm{C}$. The p-value was 0.811 , i.e. once again no significant difference exists between the examined two groups of results. These results suggest (without significance) that heterotrophic algae cultures maybe preferred for the root development of $S$. rebaudiana seedlings in soil.

The two-sample t-tests in terms of the root growth in water are presented in Fig. 9. A comparison between heterotrophic algae treatment and the control samples is shown in Fig. 9A. The p-value was 0.012 , i.e. a significant difference exists between them and heterotrophic treatment is beneficial. A contrast between heterotrophic

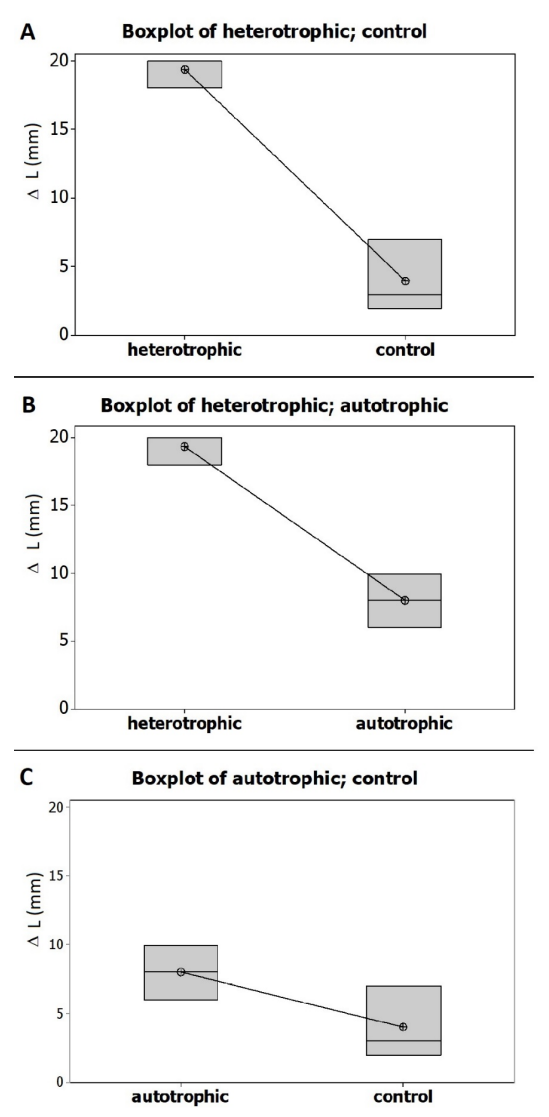

Figure 9: Box plots of two-sample t-tests in terms of root growth in water (A: heterotrophic algae vs. control, B: heterotrophic vs. autotrophic algae, C: autotrophic algae vs. control)

and autotrophic algae treatments is presented in Fig. 9B. The p-value was 0.003 , i.e. a significant difference exists between them and heterotrophic algae treatment is also beneficial. The difference between autotrophic algae treatment and the control samples is shown in Fig. 9C. The $\mathrm{p}$-value was 0.128 , i.e. no significant difference exists between them. From these results it can be concluded that while heterotrophic algae treatment was found to be significantly advantageous for the root development of $S$. rebaudiana in water, the effect of autotrophic cultures did not significantly differ from that of the control samples.

In soil a lateral-like root was formed, while in water the formation of a thicker root was observed. Just like when the root fibers were grown in land, not all the soil could be washed out without damaging the root. Therefore, a comparison between the biomass yield in water and soil was not conducted.

\section{Conclusion}

The following conclusions can be drawn from the experiments with regard to the growth of $S$. rebaudiana. Neither heterotrophic nor autotrophic algae treatments had any positive effect on the biomass growth in soil. By examining the stem growth, the autotrophic culture seemed 
to have a slightly positive effect but was not significant. In terms of root growth in soil, none of the treatments had any significant effect, but heterotrophic cultures seemed to have a slightly positive effect. Root growth in water supplemented regularly with heterotrophic algae cultures had a significant positive effect in comparison to the other treatments. Probably the positive effect of algae treatment in the case of the experiments on $S$. rebaudiana seedlings in water can be attributed to the fact that the applied algae provided complex nutrients unlike the pure tap water, while in the case of the experiments on seedlings in soil the additional nutrients provided by the algae treatment was practically negligible in comparison to those offered by the soil.

Therefore, further studies will be done to separate the effect of algae as a provider of nutrients and as a source of plant hormones.

Further studies would be necessary to prove whether changes in algae cell concentrations have an effect on the growth of Stevia rebaudiana or not, i.e. in soil, experiments on biomass growth should be applied differently in diluted algae suspensions with and without any additional nitrogen sources. Moreover, different types of light, e.g. red, blue and white, will be tested. These additional plant studies will be implemented using larger numbers of samples.

\section{REFERENCES}

[1] Geuns, J. M. C.: Stevioside. Phytochemistry, 2003 64(5), 913-921 DOI: 10.1016/S0031-9422(03)00426-6

[2] Kaur, G.; Pandhair, V.; Cheema, G. S.: Extraction and characterization of steviol glycosides from Stevia rebaudiana bertoni leaves. J. Med. Plants. Stud., 2014 2(5), 41-45 ISSN: 2320-3862

[3] Lemus-Mondaca, R.; Vega-Gálvez, A.; Zura-Bravo, L.; Kong, A. H.: Stevia rebaudiana Bertoni, source of a high-potency natural sweetener: A comprehensive review on the biochemical, nutritional and functional aspects. Food Chem., 2012 132(3) 11211132 DOI: 10.1016/j.foodchem.2011.11.140

[4] Barriocanal, L. A.; Palacios, M.; Benitez, G.; Benitez, S.; Jimenez, J. T.; Jimenez, N.; Rojas, V.: Ap- parent lack of pharmacological effect of steviol glycosides used as sweeteners in humans. A pilot study of repeated exposures in some normotensive and hypotensive individuals and in Type 1 and Type 2 diabetics. Regul. Toxicol. Pharmacol., 2008 51(1), 37-41 DOI: 10.1016/j.yrtph.2008.02.006

[5] Madan, S.; Ahmad, S.; Singh, G. N.; Kohli, K.; Kumar, Y.; Singh, R.; Garg, M.: Stevia rebaudiana (Bert.) Bertoni - A Review. Indian J. Nat. Prod. Resour., 2010 1(3), 267-286 ISBN: 3216321509, ISSN: 09760504

[6] Kinghorn, A. D. (ed.): Stevia - The genus Stevia in: Hardman, R. (ed.) Medicinal and aromatic plants Industrial profiles series Vol. 19, CRC Press, Taylor \& Francis, London UK, 2003. ISBN: 0-203-16594-2

[7] Gregersen, S.; Jeppesen, P. B.; Holst, J. J.; Hermansen, K.: Antihyperglycemic effects of stevioside in type 2 diabetic subjects. Metabolism, 2004 53(1), 73-76 DOI: 10.1016/j.metabol.2003.07.013

[8] Dyrskog, S. E. U.; Jeppesen, P. B.; Colombo, M.; Abudula, R.; Hermansen, K: Preventive effects of a soy-based diet supplemented with stevioside on the development of the metabolic syndrome and type 2 diabetes in Zucker diabetic fatty rats. Metabolism, 2005 54(9), 1181-1188 DOI: 10.1016/j.metabol.2005.03.026

[9] Puri, M.; Sharma, D.; Tiwari, A. K.: Downstream processing of stevioside and its potential applications. Biotechnol. Adv., 2011 29(6), 781-791 DOI: 10.1016/j.biotechadv.2011.06.006

[10] Kim, J.; Lee, J-Y.: Growth kinetic study of Chlorella vulgaris (August 2016), pp. 2-7. 2009. ISBN: 9781615679140

[11] Odgerel, B.; Tserendulam, D.: Effect of Chlorella as a biofertilizer on germination of wheat and barley grains Proc. Mong. Acad. Sci., 2017 56(4) 26 DOI: 10.5564/pmas.v56i4.839

[12] Faheed, F. A.: Effect of Chlorella vulgaris as biofertilizer on growth parameters and metabolic aspects of lettuce plant. J. Agri. Soc. Sci., 2008 4(4) 165-169 ISSN: 1813-2235 